\title{
Psychiater in Facharzt für psychische Erkrankungen oder für psychosoziale Medizin umbenennen - Kontra
}

\author{
Rename Psychiatrist as Specialist for Mental Diseases or as Specialist \\ for Psychosocial Medicine - Contra
}

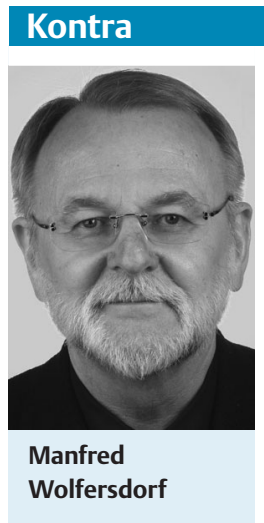

Nein, wir brauchen keine Umbenennung des psychiatrischen Facharztes „Facharzt für Psychiatrie und Psychotherapie“. Warum denn ändern? Da haben wir nun seit knapp über 200 Jahren [1-3] eine adäquate Bezeichnung dessen, was wir tun, nämlich „Psychiaterie“, eine Wortschöpfung des Visionärs Johann Christian Reil (17591813) aus den griechischen Wörtern „psyche“ für Seele und „iatro's“ für Arzt, welche die Beziehung zwischen ärztlich-medizinischem Handeln und der psychischen Situation und Problematik eines zu behandelnden Patienten anspricht. Maneros [3] weist auf Reils „Rhapsodieen“ [4] von 1803 hin, wo, im Sinne einer Antistigmakampagne, nach der Einführung der Bezeichnung „Psychiatrie“ die „Irrenhäuser“ eine neue Benennung wie „Hospital für psychische Kurmethode" bekommen und Psychiater nur bestens ausgebildete Ärzte werden sollten. 1805 war so von Johann Gottfried Langermann (1768 - 1832) (nach [5, 6]) zwei Jahre nach Erscheinen der Reilschen „Rhapsodieen“ (1803) [4] das sog. Prinzessinnenhaus, das Irrenhaus St. Georg in Bayreuth in eine „Psychische Heilanstalt für Geisteskranke“, wahrscheinlich die erste in Deutschland, umgewandelt worden.

In „Ueber den Begriff Medicin und ihre Verzweigungen, besonders in Berichtigung der Topik der Psychiaterie“, erschienen in Halle 1808, schreibt Reil [1] nicht nur bereits in der Überschrift von „Psychiaterie“, sondern betont, dass die Unterschiede zwischen den Krankheiten „bloß relative Differenz“ seien: „Daher finden wir auch niemals reine psychische, oder reine chemische oder mechanische Krankheiten, sondern in allen strahlt das Ganze wieder: Affektion des einen Le- bensprozesses der bald diese, bald jene seiner Seiten stärker herauswirft" (Reil 2008, zitiert nach [3]). Kann man dem Visionär Reil hier bereits eine ganzheitliche Betrachtungsweise zuschreiben, die überspringt man 200 Jahre - im „Common-trunk-model“ als mögliche Facharztbezeichnung „Facharzt für Psychiatrie, Psychotherapie und Psychosomatische Medizin“ oder im „Facharzt für Psychiatrie“ (verstanden im klassischen Reilschen Sinne) mit Schwerpunkten wie „Psychosomatische Medizin“ und andere endet oder gehört die „Ganzheits-Medizin“ doch Johann Christian August Heinroth (1773 - 1843) [7], dem die Schöpfung des Begriffes „psychosomatisch“ zugeschrieben wird? Neben der Arzneikunde und der Chirurgie hält Reil (2008) die Psychiatrie für einen der drei wichtigsten Hebel der Medizin. Psychiatrie ist für Reil (2008) eine medizinische Wissenschaft, Psychologie sei für die „Theorie der Psychiaterie“ fundamental.

Nun ist Reil sicher nicht der Urvater der Psychotherapie, was ja aus dem Griechischen kommend nach Kind (1986) [8] „der Seele dienend“ heißt; der Begriff soll erst im ausgehenden 19. Jahrhundert geschaffen und, so Kind (1986), erstmals von Tuka (1872) (nach [8]) in dem Buch „Illustrations of the influence of the maint upon the body“ im Kapitel „Psychotherapeutics“ verwendet worden sein. Reil (1803) [4] schreibt: „Denn es giebt noch andere Dinge, die Heilmittel sind, weil sie Krankheiten heben; aber weder chemisch, noch mechanisch, sondern psychisch wirken. Diese Kräfte, die psychisch wirken, liegen auch, wenn sie auf den bestimmten Zweck der Heilung der Krankheiten hinwirken, innerhalb der Grenze der Heilmittellehre“ (S.25) und fordert als Qualifikation für die Ausübung der „psychischen Curmethode“ „....ein vorzügliches Talent, großen Scharfblick, mehr Kenntnisse und Fähigkeiten... als jeder andere Heilkünstler, der direct auf den Körper wirkt“ ([4], S.32).
Man sieht also, der Begriff „Psychiaterie“, wenige Jahre später in „Psychiatrie“ umbenannt, beinhaltet bereits alles an psychotherapeutisch-psychiatrischer und psychosomatisch-psychiatrischer Kompetenz, was den heutigen „Psychiater“/die heutige „Psychiaterin“ ausmacht. Fassen wir zusammen: Auf den „Narrenarzt“, der nach Haisch [9] im Zusammenhang mit der Errichtung der ersten Hospitäler 15. bis 17. Jahrhundert in Deutschland durch die großen Städte und Landesherren, auftauchte - Medicus oder Kurpfuscher? -, folgte Anfang des 19. Jahrhunderts der „Psychiater“, an dessen Ausbildungsstand ein hoher Anspruch gelegt wurde. Ziel war die Humanisierung der „Irrenhäuser“, die Reil (1803) in „Heilanstalten“ umwandeln wollte zur Durchführung der „psychischen Curmethode“ - siehe seinen „Schüler“ Langermann, der in Bayreuth die wahrscheinlich erste „Psychische Heilanstalt" in Deutschland errichtete [5, 6]. Die Implementierung „der psychischen Curmethode“ als Psychotherapieform neben dem chirurgischen Eingriff oder der Pharmakotherapie; auch Fragen wie Ausbildung der Psychiater, Antistigma, Qualitätssicherung - in heutiger Sprache - oder biologische Basis der psychischen Erkrankungen zusammen mit der Relevanz psychischer und sozialer Faktoren waren wesentliche Themen $[3,4,6]$.

Warum also den „Facharzt für Psychiatrie“ bzw. „Facharzt für Psychiatrie und Psychotherapie“ (seit 1994) ändern? Ich erinnere mich an eine heftige Diskussion in der DGPPN-Mitgliederversammlung, als der Facharzt für Psychiatrie und Psychotherapie eingeführt und diskutiert wurde: Warum die Umbenennung, da „Psychiatrie“ doch die psychologisch-psychotherapeutische Dimension bereits beinhalte, wie vor 200 Jahren Reil bereits beschrieben hat. Es war letztlich eine „politische“ Entscheidung in der Diskussion mit der Psychosomatik, deren Vertreter der Psychiatrie eine einseitige biologisch-psychopharmakologische Orientierung vorwarfen (was ja bis heute nicht ganz abgeklun- 
gen ist). Verdeutlicht werden sollte durch die Einbeziehung einer Behandlungsmethode in die Facharztbezeichnung, dass der Psychiater eindeutig auch Psychotherapeut sei. Was zur obligaten, bis dahin freiwilligen, Psychotherapie-Weiterbildung der Psychiater und zu einer Implementierung psychotherapeutisch-psychosomatischer Angebote und zur Verbesserung der Reintegration von Psychotherapie in die Krankenhauspsychiatrie führte.

Jeder zusätzlich eingeführte Begriff, wie z.B. „psychosozial“ oder „psychosomatisch“ in die klassische Facharztbezeichnung würde wieder zu einer ähnlichen Diskussion führen und letztendlich dann den Begriff „Psychiatrie“ doch ganz gegen den ursprünglichen Sinn reduzieren auf biologische Aspekte, Hardcore-Psychiatrie, auf die sog. schweren Kernformen von Psychiatrie aus dem Feld der Suchtkrankheiten, der demenziellen Erkrankungen, der chronischen Psychosen und den Eindruck vermitteln, als bräuchte die Psychiatrie zur Erfüllung ihres Behandlungsauftrags entlang der gesamten ICD10 zusätzliches Beiwerk, zusätzliches Handwerkszeug, neue Orientierung. Natürlich wissen wir alle, dass es innerhalb der Psychiatrie unterschiedliche Schwerpunkte gibt, die dann auch in unterschiedlichen Bezeichnungen immer wieder in der Literatur geführt werden, von der „biologischen Psychiatrie“ über die „Sozialpsychiatrie“ bis hin zur „psychodynamischen Psychiatrie“ usw. Aber wir wissen auch, dass dies unter dem „Dach Psychiatrie“" geschieht.

Nun gibt es in Deutschland neben dem Facharzt für Psychiatrie und Psychotherapie auch den Facharzt für Psychosomatische Medizin und Psychotherapie und es gibt seit Ende der 1990er-Jahren die Diskussion um einen „Common trunk“. Würden wir also den Facharzt für Psychiatrie und Psychotherapie ergänzen um Psychosomatische Medizin, würde das wie schon ausgeführt die Benennung Psychiatrie, obwohl sie dies alles beinhaltet, weiter abwerten. Das „Common-trunk-model“, vor über einem Dutzend Jahren vom DGPPN-Vorstand heftig vertreten, mit einem „Facharzt für Psychiatrie“ als ge- meinsames Dach und Schwerpunktbezeichnungen wie „Psychosomatische Medizin“ oder „Psychotherapie“ oder „Forensische Psychiatrie“ u. Ä. wäre adäquat, inhaltlich korrekt und würde die Wiedereinbindung von Psychosomatik und Psychotherapie unter dem gemeinsamen Dach der Psychiatrie ermöglichen.

Die Umbenennung von „Nervenkrankenhäusern“ der 50er- und 60er-Jahre in Kliniken für Psychiatrie, dann weiter in Kliniken für Psychiatrie, Psychotherapie und Psychosomatik hat zumindest zu einer Annährung, vielleicht sogar Gleichstellung mit somatischen Kliniken geführt und die psychotherapeutische Kompetenz für die Bevölkerung betont - bis heute ein Dissensthema mit der Psychosomatik. Klinken für Chirurgie oder Dermatologie benennen das Gebiet, nicht die Erkrankung. Auch wir sollten dabei bleiben. Ich glaube nicht, dass sich Stigmatisierung psychisch kranker Menschen durch Umbenennung der Behandler und der Kliniken wesentlich reduzieren lässt; weil Stigma auf den Kranken und das Nichtverstehen von psychischer Krankheit zielt. Und die ändern sich ja nicht.

Die Einführung des Facharztes „Facharzt für Psychiatrie und Psychotherapie“ war eine Kompromissbildung bzw. eine Anpassungsleistung im Rahmen ökonomischer, Abgrenzungs- und vor allem Antistigmabemühungen von DGPPN, BDK und Abteilungspsychiatrie. Ein wiederbelebtes „Common-trunk-model“, unter dem gemeinsamen Dach „Psychiatrie“, verstanden im klassischen Reilschen Sinne, ausgehend von einem gemeinsamen Weiterbildungsstamm, die Benennung von Schwerpunkten erlaubend, würde die elendige Auseinandersetzung zwischen den „Psych“-Gruppen zumindest auf der Ebene der Facharztbezeichnung beenden. Aus meiner Sicht brauchen wir keine Umbenennung des psychiatrischen Facharztes, sondern eine Wiederbelebung der Diskussion um das „Common-trunkmodel“.

Sie haben eine eigene Meinung zu diesem Thema? Dann schreiben Sie uns an: psychiat-praxis@thieme.de!

\section{Literatur}

1 Reil JC. Ueber den Begriff Medicin und ihre Verzweigungen, besonders in Beziehung auf die Berichtigung der Topik der Psychiaterie. Halle: 1808

2 Marneros A, Pillmann F. Das Wort Psychiatrie wurde in Halle geboren. Von den Anfängen der deutschen Psychiatrie. Halle: Fliegenkopf; 2004

3 Maneros A. Die Geburtsstunde der psychiatrischen Wissenschaft und Heilkunde in Deutschland. Die Psychiatrie 2004; 1: 1-8

4 Reil JC. Rhapsodieen über die Anwendung der psychischen Curmethode auf Geisteszerrüttungen. Halle: 1803

5 Aas $N$. „Es ist ausgemacht, dass sich zur Heilung der Wahnsinnigen ungleich mehr beitragen lässt, als bisher in dem Baireuther Irrenhause und in noch vielen anderen ähnlichen Instituten geschehen ist." Vom Irrenhaus zur Irren-Heilanstalt St. Georgen (1788-1810). In: Wolfersdorf M, Ettle M, Moos M, Purucker M, Hrsg. Psychiatrie in Bayreuth. Regensburg: Roderer; 2005: $35-$ 70

6 Wolfersdorf M. 200 Jahre „Psychische Heilanstalt" Bayreuth. Anmerkungen zur Entwicklung der klinischen Psychiatrie und Psychotherapie in Bayreuth. In: Wolfersdorf M, Ettle M, Moos M, Purucker M, Hrsg. Psychiatrie in Bayreuth. Regensburg: Roderer; 2005: 9-34

7 Steinberg H. Johann Christian August Heinroth (1773-1743) - der erste Lehrstuhlinhaber für Psychiatrie und sein Krankheitskonzept. In: Angermeyer MC, Steinberg H, Hrsg. 200 Jahre Psychiatrie an der Universität Leipzig. Personen und Konzepte. Heidelberg: Springer Medizinverlag; 2005: 1-80

8 Kind H. Psychotherapie. In: Müller Chr, Hrsg. Lexikon der Psychiatrie. 2. Auflage. Berlin, Heidelberg, New York: Springer-Verlag; 1986: $568-571$

9 Haisch E. Irrenpflege in alter Zeit. CIBA-Zeitschrift 1959; 95: 3142 -3171, insb. S. 3143

Korrespondenzadresse

Prof. Dr. med. Dr. h. c. Manfred Wolfersdorf Bezirkskrankenhaus Bayreuth/

Bezirksklinik Hochstadt

Nordring 2

95445 Bayreuth

manfred.wolfersdorf@bezirkskrankenhausbayreuth.de

\section{Bibliografie}

DOI http://dx.doi.org/

10.1055/s-0033-1349616

Psychiat Prax 2014; 41: 13-14

(c) Georg Thieme Verlag KG

Stuttgart - New York

ISSN 0303-4259 